\title{
Characterization of Macrolesions Induced by Myocardial Cavitation-Enabled Therapy
}

\author{
Yiying I. Zhu, \\ Biomedical Engineering, University of Michigan, Ann Arbor, MI 48109 USA \\ Douglas L. Miller, \\ Department of Radiology, University of Michigan Medical Center, Ann Arbor, MI 48109 USA \\ Chunyan Dou, and \\ Department of Radiology, University of Michigan Medical Center, Ann Arbor, MI 48109 USA \\ Oliver D. Kripfgans [Member IEEE] \\ Department of Radiology, University of Michigan Medical Center, Ann Arbor, MI 48109 USA
}

Yiying I. Zhu: zhuyiy@umich.edu; Douglas L. Miller: douglm@umich.edu; Chunyan Dou: chunyand@med.umich.edu; Oliver D. Kripfgans: oliver.kripfgans@umich.edu

\section{Abstract}

Intermittent high intensity ultrasound pulses with circulating contrast agent microbubbles can induce scattered cavitation myocardial microlesions of potential value for tissue reduction therapy. Here, computer-aided histological evaluation of the effective treated volume was implemented to optimize ultrasound pulse parameters, exposure duration, and contrast agent dose. Rats were treated with $1.5 \mathrm{MHz}$ focused ultrasound bursts and Evans blue staining indicates lethal cardiomyocytic injury. Each heart was sectioned to provide samples covering the entire exposed myocardial volume. Both brightfield and fluorescence images were taken for up to 40 tissue sections. Tissue identification and microlesion detection were first done based on 2D images to form microlesion masks containing the outline of the heart and the stained cell regions. Image registration was then performed on the microlesion masks to reconstruct a volume-based model according to the morphology of the heart. The therapeutic beam path was estimated from the 3D stacked microlesions, and finally the total microlesion volume, here termed macrolesion, was characterized along the therapeutic beam axis. Radially symmetric fractional macrolesions were characterized via stepping disks of variable radius determined by the local distribution of microlesions. Treated groups showed significant macrolesions of a median volume of $87.3 \mu \mathrm{L}, 2.7$ $\mathrm{mm}$ radius, $4.8 \mathrm{~mm}$ length, and $14.0 \%$ lesion density compared to zero radius, length, and lesion density for sham. The proposed radially symmetric lesion model is a robust evaluation for Myocardial Contrast Enabled Therapy (MCET). Future work will include validating the proposed method with varying acoustic exposures and optimizing involved parameters to provide macrolesion characterization. 


\section{Index Terms \\ Cavitation microlesions; cavitation therapy; hypertrophic cardiomyopathy; myocardial macrolesion; therapeutic ultrasound}

\section{Introduction}

Hypertrophic cardiomyopathy (HCM), involving enlargement and hyperplasia of the myocardium, occurs in at least one out of 500 people. Sudden death is the most visible and unpredictable consequence of HCM, occurring without warning signs or symptoms. It has been reported that HCM is the most common cause of sudden death in young people, and is the most frequent cause of sudden death in US competitive athletes [1]. Although pharmacologic therapies have exhibited significant positive effects, there remain substantial undesired side effects, leaving approximately a third of patients as candidates for myocardial reduction [2].

The definitive treatment for HCM is surgical myectomy [3], which involves resection of a small portion of the interventricular septum at its base. Less-invasive alternatives have been explored using a variety of techniques, most commonly an endovascular procedure.

However, the need for permanent pacing, the inability to address concomitant anatomic abnormalities and the uncertainty about the long-term effects of having caused substantial myocardial necrosis and scaring are undesirable. Other approaches such as thermal ablation [4] or histotripsy [5] reduce cardiac tissue by accumulating ultrasonic focal lesions.

For decades, ultrasonic bioeffects from microbubble inertial cavitation have been discussed for their therapeutic potentials [6]. Concurrent studies have investigated cavitation-induced microvessel and cell injuries [7]. A novel technique using contrast echocardiography using higher than diagnostic pressure amplitudes, named Myocardial Contrast Enabled Therapy (MCET), is hypothesized to induce a fractional macrolesion with sparse and histologically definable microlesions, which in turn shrink the diseased heart muscle without substantial scaring [8].

Currently, therapeutic ultrasound has great potential in medicine as a noninvasive method to treat tumors, promote hemostasis, and treat other diseases due to its ability to penetrate deeply and deposit thermal or mechanical energy at a specific site with submillimeter accuracy. Various imaging modalities, including x-ray, computed tomography, magnetic resonance imaging, and ultrasound imaging, can provide precise targeting and therapy monitoring [9].

Passive cavitation imaging has been demonstrated for potential use with continuous-wave high-intensity focused ultrasound thermal ablation [10] and for pulsed-wave ultrasound therapy insonations [11]. A system for microbubble-mediated sonothrombolysis utilizing therapy guidance and cavitation imaging has been developed [12].

MCET is performed under the guidance of echocardiography. Ventricular premature complexes and blood pressure can provide temporal feedback [13], while inertial cavitation 
mapping combined with anatomical imaging could delineate the spatial accumulation of cardiomyocytic response.

To evaluate the effectiveness of MCET treatment and modulate its treatment parameters, such as ultrasound amplitude, contrast dose, and treatment duration, an evaluation scheme is needed for characterizing microlesions. In previous studies, myocardial necrosis [8][14] was evaluated qualitatively by visual identification and scoring of Evans blue-stained cells in frozen histological slices [15]. However, visual scoring only yields a limited onedimensional descriptor, i.e. scalar information of damaged cells assessed for each tissue slice, which is far from adequate to describe a comprehensive treatment outcome for tuning therapeutic inputs. For example, a qualitative score of 58,790 more than 23,560 helped making relative judgments about the optimum exposure parameters, but it gave no information about the amount of tissue reduction. Thus, objective and quantitative scoring is needed to precisely determine treatment outcome and volume-based treatment spatial distribution evaluation is essential to ultimately develop a safe and effective therapeutic application.

Numerous methods have been devised for cellular and histological analysis. Molecular imaging, defined as the visual representation, characterization, and quantification of biological processes at cellular and subcellular levels, could be used to non-invasively detect and monitor cancer treatments [16]. It enables tumor localization, spatial visualization of specific molecular markers, and biological processes that influence tumor behavior and/or response to therapy [17].

Regarding cellular microscopy, computational imaging provides multidimensional and quantitative image analysis, enabling mathematical modeling of cell biology. Employed process steps include image acquisition and pre-processing, registration, segmentation, volume rendering, et cetera [18]. Image segmentation, including thresholding, region growing, and clustering, plays an important role in characterizing contents of medical images [19].

This paper describes a scheme for MCET characterization in acute preclinical studies. A computer-aided histological evaluation of MCET-induced macrolesions has been implemented to quantify treatment outcome. The point of the presented method is to provide the volumetric macrolesion determination to approximate the expected amount of tissue reduction, which was not available from the qualitative visual score. Two-dimensional brightfield and fluorescence images were evaluated by an algorithm, which automatically identified microlesions, and characterized the volume-oriented fractional macrolesion. Subsequently, this method can be applied to optimize ultrasound pulse parameters, exposure duration and contrast agent dose for MCET treatment of hypertrophic cardiomyopathy, which would require up to $25 \%$ vol-vol tissue reduction in some myocardial areas. 


\section{Method}

\section{A. Animal and Tissue Preparation}

In concomitant research designed to optimize the timing of pulses for myocardial cavitationenabled therapy, tissue samples were collected and prepared for histological evaluation [13]. For this study, these samples were utilized for development of computer-aided evaluation. Briefly, in vivo animal procedures were conducted on twenty male Sprague-Dawley rats (Charles River, Wilmington, MA, USA) and five sham rats weighing $331 \pm 33 \mathrm{~g}$ under the approval and guidance of the University Committee on Use and Care of Animals. All rats in the treated group were injected with Definity ${ }^{\circledR}$ (Lantheus Medical Imaging, Inc., N.

Billerica, MA) at a rate of $5 \mu \mathrm{L} / \mathrm{kg} / \mathrm{min}$. MCET was performed with $1.5 \mathrm{MHz}$ ultrasound burst of five cycle pulses at 4.0 MPa peak rarefactional pressure amplitude by use of a 1.9 $\mathrm{cm}$ diameter and $3.8 \mathrm{~cm}$ focus single element therapy transducer, triggered at one beat out of four heart beats. Before being exposed to 5 minutes of therapy, all rats were injected with Evans blue, which has been proven to be a dependable stain for histological determination of cell necrosis [20].

After being frozen in compound (Tissue-Tek, Sakura Finetek USA Inc. Torrance CA USA) on dry ice, each heart was dissected to provide samples covering the entire exposed myocardial volume. Up to thirty sections, $10 \mu \mathrm{m}$ thick, for each heart were cut every $200 \mu \mathrm{m}$ into the sample to cover about $5 \mathrm{~mm}$ of the dissected portion of the heart. Two-hundred micrometer spacing was set to avoid possible overlap of stained cardiomyocytes in adjacent sections and thoroughly sample the cardiac volume. However, it should be noted that some stained cells present in between sections were not actually observed, but assumed by extrapolation to have the same fractional lesion area over the $190 \mu \mathrm{m}$ unsampled space.

\section{B. Cardiomyocyte Scoring}

Besides the proposed automatic characterization scheme, visual scoring and measurement of troponin I in plasma were performed to evaluate cardiomyocyte staining. Visual scoring was based on Evans blue staining as described previously [13]. Troponin I was analyzed from plasma samples collected one day after euthanasia with an ELISA assay kit (Rat Cardiac TnI (ultra sensitivity), Life Diagnostics Inc. West Chester PA USA) as described previously [13].

\section{Microscope Image Acquisition}

To enable computer aided evaluation, both brightfield and fluorescence images were obtained for each tissue-section through a microscope camera system (SPOT Flex, Diagnostic Instruments Inc., Sterling Heights MI USA) via fluorescencent stereomicroscopy (Leica MZ FLIII, Leica Microscopy Systems Ltd., Heerbrugg, Switzerland) with a highresolution and large field of view objective (Leica Plan APO 1.6x, Leica Microsystems Ltd., Heerbrugg, Switzerland). The images were acquired as 16 bit 4096 by 4096 RGB, covering the entire tissue sections. One image set of treated myocardium at the approximate midpoint of the treated zone is shown in Fig. 1(a) and Fig. 1(b); a sham image set is shown in Fig. 1(c) and d. Macrolesion projection in 2D, noted by MaL in Fig. 1a, was defined as a bulk of the microlesions within the target area; MaL identification is described below. Left ventricle 
$(\mathrm{LV})$, right ventricle (RV), and septum (SP) are also labeled. The damaged cardiomyocytes in scattered microlesions appear stained light blue in the brightfield image, together with erythrocytes and other cells associated with the co-located microvascular hemorrhage, as shown in Fig. 1(a). The damaged cardiomyocytes specifically appear fluorescent red in the relatively high contrast fluorescence image as shown in Fig. 1(b). A microlesion was defined simply as a local collection of one or a few damaged cardiomyocytes.

In contrast, normal heart tissue appears grayer in the brightfield image and darker in the fluorescence image. The therapeutic ultrasound beam path projected onto the $2 \mathrm{D}$ image is estimated from the lesion cloud as shown in Fig. 1(b). Brightfield images were taken using auto exposure to provide morphologic information and fluorescence images were taken using a constant exposure to provide constant and quantitative lesion contrast information.

\section{Cardiomyocyte Modeling}

An average cross-sectional area $\left(A_{C}\right)$ for one cardiomyocyte sliced in a tissue section was estimated based on a mathematical model, which then gave an approximation of the number of stained cells by dividing the total injured area by the estimated $A_{C}$. A cardiac myocyte cell was geometrically modeled as an elliptic cylinder with cell dimensions summarized in Table 1 based on previous work [21]. The left ventricular wall comprises three strands of different longitudinal alignment: superficial (subepicardial) noted as $\mathrm{S}$, middle noted as $\mathrm{M}$, and deep (subendocardial) noted as D. The myocyte model approximated the superficial 'layer' at an angle of $15^{\circ}$ with respect to the long axis of the left ventricular inlet, occupying $25 \%$ of the wall thickness, the middle 'layer' circumferentially arranged occupying $56 \%$ of the wall thickness, and the deep 'layer' radiating longitudinally accounting for $19 \%$ of the wall thickness [22].

As illustrated in Fig. 2, a slice plane intersects with layers of different cell orientation, resulting in variable cross-sectional areas. The average cross-sectional cell area $A_{C}$ is then given by

$$
A_{C}=\bar{A}_{S} P_{S}+\bar{A}_{M} P_{M}+\bar{A}_{D} P_{D}
$$

where $\bar{A}_{S}, \bar{A}_{M}$ and $\bar{A}_{D}$ are average areas for the three layers: $S, M$ and $D . P_{S}, P_{M}$ and $P_{D}$ are the fractional volume percentages of each layer for the overall wall thickness, namely $P_{S}$ $=25 \%, P_{M}=56 \%$ and $P_{D}=19 \%$.

For the superficial layer, we assumed that the cut-off plane did not reach the edge so that the cross-section was always strictly an ellipse. Thus, cross-section areas for superficial and deep layers were both considered as ellipses, (2) and (3) respectively,

$$
\bar{A}_{S}=\pi \cdot \frac{w}{2 \cos 15^{\circ}} \cdot \frac{d}{2 \cos 15^{\circ}},
$$




$$
\bar{A}_{D}=\pi \cdot \frac{w}{2} \cdot \frac{d}{2} .
$$

For the middle layer, the intersection plane was always a rectangle with length equal to $l$, and width $G H$ as depicted in Fig. 3 and determined by the orientation that the cutting plane intersects with the elliptic cylinder (4)

$$
\frac{x^{2}}{w^{2}}+\frac{y^{2}}{d^{2}}=1
$$

with $-w \leq x \leq w,-d \leq y \leq d$, intersects with the cutting line (5)

$$
y=\tan \theta \cdot x+h
$$

with $0 \leq x \leq \pi / 2,0 \leq y \leq h_{\max }(\theta)$ at points $G(x 1, y 1)$ and $H(x 2, y 2)$, if and only if $x, y$ have real solutions. For a certain cutting angle $\theta$, the maximum value that $h$ could achieve is when the cutting line is tangent to the ellipse. Thus, $h_{\max }$ is a function of $\theta$. Thus, the length of the cutting edge $G H, L_{G H}$, is a function of $\theta$ and $h$ as in (6)

$$
L_{G H}(h, \theta)=\sqrt{\left(x_{1}(h, \theta)-x_{2}(h, \theta)\right)^{2}+\left(y_{1}(h, \theta)-y_{2}(h, \theta)\right)^{2}} .
$$

We assume that $\theta$ and $\mathrm{h}$ are uniformly distributed variables within their range, i.e.

$$
\begin{gathered}
p(\theta)=\left\{\begin{array}{c}
\frac{2}{\pi}, \\
0 \leq \theta \leq \frac{\pi}{2}, \\
0, \\
\frac{\pi}{2}<\theta<2 \pi ;
\end{array}\right. \\
p(h)=\left\{\begin{array}{c}
\frac{1}{h_{\max }(\theta)}, \\
0 \leq h \leq h_{\max }(\theta), \\
0, \\
\text { otherwise. }
\end{array}\right.
\end{gathered}
$$

where $\mathrm{p}$ indicates the probability density function for $\mathrm{p}(\theta)$ and $\mathrm{p}(\mathrm{h})$. The average crosssectional area $\bar{A}_{M}$ in this case is (9)

$$
\bar{A}_{M}=l \cdot \bar{L}_{G H}
$$

where

$$
\begin{aligned}
& \bar{L}_{G H}=\int_{\theta, h} L_{G H}(h, \theta) P(h, \theta) d h d \theta \\
& =\int_{0}^{\pi / 2} \int_{0}^{h_{\max }(\theta)} L_{G H}(h, \theta) P(h) P(\theta) d h d \theta .
\end{aligned}
$$


Finally, given the above relationships, an average cross-section area, $\bar{A}_{C}$, of $2399 \mu \mathrm{m}^{2}$ can be computed. The pixel size for all 2D digital images is $15.7 \mu \mathrm{m}^{2}$. Thus, one cell occupies approximately $2399 \mu \mathrm{m}^{2} / 15.7 \mu \mathrm{m}^{2} /$ pixel, that is 153 pixel in the acquired images. An approximation for the number of stained cardiomyocytes could then be inferred.

\section{E. Lesion Characterization}

To reduce computation complexity and save computation memory, the red channel, of all the data was first extracted. All the images were preprocessed to remove uneven illumination and zero padded ready for registration before feeding into the lesion characterization processing. Background illumination for both brightfield images and fluorescence images were estimated using a Gaussian low-pass filer [23], which was then subtracted from the original images. The normalized images were processed as shown in Fig. 4. The main steps to characterize macrolesions include: 1) tissue detection, 2) microlesion detection, 3) image registration and 3D stacking, 4) therapeutic beam estimation, and 5) macrolesion characterization.

Tissue detection and microlesion detection were first done based on 2D images to form microlesion masks, which contain the morphologic information of the heart as well as stained cell regions. Image registration was then performed on microlesion masks to reconstruct a volume-based model according to the morphology of the heart. The therapeutic beam was estimated from the 3D stacked microlesions, and finally a macrolesion was characterized along the therapeutic beam.

Step 1: Tissue Detection-The main objective in this step is to segment the tissue portion based on morphologic information from brightfield images in order to constrain the subsequent characterization. Brightfield images (see Fig. 5(a) were downsampled first to be segmented and then upsampled back after segmentation to decrease computational load. A threshold mask as shown in Fig. 5(b) and an edge-detected mask as shown in Fig. 5(c) were obtained simultaneously from a normalized brightfield image as the example shown in Fig. 5(a), which was then fused together giving the tissue mask as shown in Fig. 5(d).

Step 2: Microlesion Detection-Starting with an initial guess of $10 \%$ for the lesion density, two lesion masks were obtained from thresholding brightfield and fluorescence images respectively. An automatic threshold, described below, for the fluorescence image was then determined from the statistics of pixel values within the mutual mask of the two lesion masks. An example of a lesion mask on top of a zoomed original brightfield image Fig. 6(a) is shown in Fig. 6(b) illustrating the morphologies of the detected lesions.

Step 3: Image Registration and 3D Stacking-In order to process volume-based characterization, 2D lesion masks were stacked to $3 \mathrm{D}$ according to the information provided through neighboring brightfield images from the tissue slide stack, i.e. by means of the transformation information from rigid image registration. Stacking registered mask images allows for visualization of the employed 3D model as shown in Fig. 7, where the red contour is rendering the tissue edges as resulting from step 1, black marks symbolize microlesions detected in step 2. For the purpose to visualize the overall effect of treatment, high- 
resolution image showing the detailed morphology of individual cell is unnecessary, thus 32 times downsampled microlesions symbolized by black marks are displayed representing the trend of lesion density. However, the original resolution is retained for later characterization. The inner red oval shape is the exclusion representing the inner surface of the left ventricle.

Step 4: Therapeutic Beam Estimation-The therapeutic beam vector shown as a blue line with an arrow in Fig. 7 was characterized by least square line fitting from the detected microlesions, excluding auto-fluorescing blood vessels and other non-microlesion microstructures, which were considered as noise.

Step 5: Macrolesion Characterization-The stacked 3D heart model was re-sliced along the therapeutic beam in $800 \mu \mathrm{m}$-thick steps. Note that the re-slices here are not tissuesections but slices perpendicular to the identified ultrasound beam. At each position along the beam axis, a disk was characterized as illustrated in Fig. 8(a): each such slice was first populated with a $20 \mathrm{~mm}$-diameter disk coaxial to the beam; each disk was then progressively reduced in size until a certain microlesion quantity was reached from a dynamic threshold, the criterion of which is explained below. Disks were characterized individually and then stacked together resulting in a final macrolesion as shown in Fig. 8(b).

To determine the dynamic radius mentioned above, a dynamic percentage value of overall stained cells within a maximum slice volume is computed. The threshold is a piecewise function of total number of stained cells and is shown in Fig. 9. Here:

$$
\begin{aligned}
& \text { if } \mathrm{N} \geq \mathrm{N}_{0}, \mathrm{~T}=\mathrm{T}_{0} ; \\
& \text { if } \mathrm{N}<\mathrm{N}_{0}, \mathrm{~T}=\max \left(\mathrm{T}_{0}(1-\exp (-\mathrm{N})), \mathrm{T}_{0}\right),
\end{aligned}
$$

where $\mathrm{N}$ indicates the total number of stained cells detected, $\mathrm{N}_{0}$ is a cut-off threshold for piece-wise function; and $\mathrm{T}$ indicates the percentage threshold for radius selecting with a constant $\mathrm{T}_{0}$ as a maximum. As illustrated in Fig. 9, the example here sets $\mathrm{N}_{0}=30, \mathrm{~T}_{0}=$ $95 \%$.

The in vivo point spread function (PSF) of the therapeutic beam was characterized as a function of space by first sampling along the therapeutic beam interrogating co-axial cylinders of $\lambda(1 \mathrm{~mm})$ diameter and quarter $\lambda$ length. Then, at the point of maximum axial lesion density, the lateral direction was sampled with co-axial rings of one-eighth $\lambda$ width and quarter $\lambda$ height.

\section{Results}

\section{A. Lesion Visualization}

A radially symmetric macrolesion was characterized via stepping disks of various radius determined by the local distribution of microlesions.

An example is shown in Fig. 10. An example of a 3D macrolesion intersecting with one of the original 2D microscopy images is shown in Fig. 11 with a delineated boundary indicating macrolesion projection and tissue boundary characterized from brightfield tissue detection. 


\section{B. Group Comparison}

As described in a concomitant study [13], the treated group of rats was subdivided into three groups, with five rats in each group. They were treated at three different time points of the cardiac cycle: at the onset of $\mathrm{R}$ wave ( $\mathrm{RR}$, end diastole), at the $\mathrm{R}$ wave plus one third of the $R$ to $R$ interval ( $R R / 3$, end systole) and at the $R$ wave plus two thirds of $R R(2 R R / 3$, middiastole). Results for macrolesions characterized for all 20 rats are presented in Fig. 12 using boxplots. For each box, the central mark is the median, the edges of the box are the $25^{\text {th }}$ and $75^{\text {th }}$ percentiles, the whiskers extend to the most extreme data points not considered outliers, and outliers are plotted individually. The normal range was defined as $\mathrm{q} 3+1.5$ (q3 - q1) or smaller than $\mathrm{q} 1-1.5(\mathrm{q} 3-\mathrm{q} 1)$, where $\mathrm{q} 1$ and $\mathrm{q} 3$ are the $25^{\text {th }}$ and $75^{\text {th }}$ percentiles, respectively. Corresponding median values for treated groups are reported in Table 2 . The results show no significant variation between treated groups, which agree with the visual scoring results from the concomitant study.

The summarized results for the treated group of fifteen rats compared to sham group shows significant macrolesions with a median volume of $87.3 \mu \mathrm{L}$ of $14.0 \%$ lesion density, $2.7 \mathrm{~mm}$ macrolesion radius and $4.8 \mathrm{~mm}$ macrolesion length compared to zero lesion density, radius and length for the sham group. This overall result was consistent with the measurements of troponin, which were also indicative of total cardiomyocyte necrosis.

Point Spread Function-The in vivo point spread function (PSF) of the therapeutic beam in terms of lesion density was characterized for one rat affected by an extreme long therapeutic path through the myocardium, as shown in Fig. 13.

\section{Discussion}

This computer aided histological evaluation appears to provide robust characterization of the macrolesion formed by the accumulation of microlesions based on the following considerations.

\section{A. Therapeutic Beam Estimation}

Occurrence of cavitation events induces the damage of myocardial cells. Assuming contrast agent in the myocardium is uniformly distributed, the fraction volume percentage is dominated by the acoustic beam profile. Above the cavitation threshold, the likelihood of cavitation increases as the acoustic pressure increases under acoustic pressure present in our experiment. We expect the density trend of the microlesion somewhat agrees with the acoustic beam profile, but not exactly because the formation of the lesion is an accumulation consequence and the process involves the biological activity of myocardium. For example, breathing motion leads to smearing out the accumulation effect of the treatment, which then is not shaped strictly according to therapeutic beam. Thus in turn, the therapeutic beam estimation is affected. A decreasing trend of the lesion density was observed along the axial direction, possibly due to acoustic attenuation caused by contrast agents in the left ventricle. The lateral PSF profile was obtained at the maximum of the axial response, which was regarded as the volume of interest of the therapy. The $-6 \mathrm{~dB}$ lateral width of roughly $2.8 \mathrm{~mm}$ 
agrees reasonably with $3.5 \mathrm{~mm}$ beam diameter of transducer in free-field considering propagation attenuation in tissue and cavitation as a threshold event.

\section{B. Cardiomyocyte Model Validation}

A total of 160 manually selected cells according to the morphology appearing in brightfield images were taken an average to obtain estimation of area for a cell of $2719 \mu \mathrm{m}^{2}$ versus $2398 \mu \mathrm{m}^{2}$ from the theoretical geometric model. The $12 \%$ deviation of the estimation model from the selected estimation comes from bias on selecting large identifiable cells on the manual side and the variation from the cell orientation from the modeling side.

\section{Cardiomyocyte Cell Scoring Validation}

The Evans blue staining found in tissue frozen after one day identifies lethally injured cells forming microlesions [8][9]. Therefore, the total determination of the macrolesion as a fraction of the tissue volume provides a first approximation to the amount of tissue reduction expected from the treatment. The desired reduction for therapeutic treatment would be about $20 \%$, which could be expanded beyond the single focus treatment described here by moving the beam to provide the needed therapeutic reduction in larger (i.e. human) hearts. The simple visual scoring of the stained cells provide only a qualitative measure of the microlesion effect, particularly for high densities of microlesions seen in the treatments intended for therapeutic tissue reduction, which only approximate the more accurate image analysis values (Fig. 13). In addition, the qualitative visual scores fail to give a total fractional reduction throughout the treated volume, which is provided by the reconstruction of the volume and the macrolesion determination.

The cell identification scheme counts pixels rather than cells assuming statistical mean for cell size with respect to pixels, by which the counting would not oversample the cells because of their complicated morphology. The automatic scheme of stained cell identification seems reasonable compared to visual scoring as shown in Fig. 14. The cases of large accumulations of stained cells were often difficult to score visually resulting in qualitative results rather than actual cell count. In contrast, the proposed computer-based characterization method is objective and quantitative and overcomes the limitation of large number of cells.

\section{Lesion Characterization Criterion}

The volume estimation of macrolesions considered local lesion density as well as total lesion number. Additionally, a dynamic radius cutoff criterion was used that depends on the number of the stained cells. Using a constant threshold for stained cell intensity would not affect the shape of a well-generated dense lesion due to the large number of microlesions in the macrolesion volume, but would affect a sparse macrolesion due to its low number density of microlesions. Thus, a dynamic threshold was introduced.

\section{Conclusion}

A computer-aided three-dimensional objective evaluation scheme has been developed to characterize macrolesions, including lesion size and lesion density, for MCET to reduce 
myocardial tissue, based on brightfield and fluorescence images as available in acute preclinical studies. The radially symmetric model employed to characterize macrolesion density is feasible for the study using a single focused beam. This methodology reduces visual scoring ambiguity and provides a volume oriented, quantity-sensitive therapy evaluation. The significance of characterized macrolesion compared to sham group demonstrates that the evaluation scheme is robust against noise. Future implementations of MCET involve formulating a set of optimized treatment parameters to create a desired total volume reduction based on lesion density within the target volume. And the proposed evaluation scheme would possibly assist other therapeutic applications resulting in sparse effect, such as drug delivery, gene therapy and blood brain barrier opening.

\section{Acknowledgments}

This work was supported in part by National Institutes of Health under Grant 1-R01-HL-114595-01.

\section{References}

1. Maron BJ, Maron MS. Hypertrophic cardiomyopathy. Lancet. Jan 19.2013 381:242-55. [PubMed: 22874472]

2. Marian AJ. Contemporary treatment of hypertrophic cardiomyopathy. Tex Heart Inst J. 2009; 36:194-204. [PubMed: 19568388]

3. Ommen SR. Hypertrophic cardiomyopathy. Curr Probl Cardiol. Nov.2011 36:409-53. [PubMed: 21962729]

4. Otsuka R, Fujikura K, Abe Y, Okajima K, Pulerwitz T, Engel DJ, et al. Extracardiac ablation of the left ventricular septum in beating canine hearts using high-intensity focused ultrasound. J Am Soc Echocardiogr. Dec.2007 20:1400-6. [PubMed: 17588712]

5. Xu Z, Ludomirsky A, Eun LY, Hall TL, Tran BC, Fowlkes JB, et al. Controlled ultrasound tissue erosion. IEEE Trans Ultrason Ferroelectr Freq Control. Jun.2004 51:726-36. [PubMed: 15244286]

6. Miller DL, Averkiou MA, Brayman AA, Everbach EC, Holland CK, Wible JH, et al. Bioeffects considerations for diagnostic ultrasound contrast agents. J Ultrasound Med. Apr.2008 27:611-632. [PubMed: 18359911]

7. Chen H, Brayman AA, Evan AP, Matula TJ. Preliminary Observations on the Spatial Correlation between Short-Burst Microbubble Oscillations and Vascular Bioeffects. Ultrasound Med Biol. Dec. 2012 38:2151-2162. [PubMed: 23069136]

8. Miller DL, Li P, Gordon D, Armstrong WF. Histological characterization of microlesions induced by myocardial contrast echocardiography. Echocardiography. Jan.2005 22:25-34. [PubMed: 15660683]

9. Vaezy S, Andrew M, Kaczkowski P, Crum L. Image-guided acoustic therapy. Annu Rev Biomed Eng. 2001; 3:375-90. [PubMed: 11447068]

10. Gyongy M, Coussios CC. Passive spatial mapping of inertial cavitation during HIFU exposure. IEEE Trans Biomed Eng. Jan.2010 57:48-56. [PubMed: 19628450]

11. Haworth KJ, Mast TD, Radhakrishnan K, Burgess MT, Kopechek JA, Huang SL, et al. Passive imaging with pulsed ultrasound insonations. J Acoust Soc Am. Jul.2012 132:544-553. [PubMed: 22779500]

12. Vignon F, Shi WT, Powers JE, Everbach EC, Liu JJ, Gao SJ, et al. Microbubble Cavitation Imaging. IEEE Trans Ultrason Ferroelectr Freq Control. Apr.2013 60:661-670. [PubMed: 23549527]

13. Miller DL, Dou C, Owens GE, Kripfgans OD. Optimization of ultrasound parameters of myocardial cavitation microlesions for therapeutic application. Ultrasound Med Biol. 2014; 40:1228-36. [PubMed: 24613640] 
14. Miller DL, Li P, Dou C, Gordon D, Edwards CA, Armstrong WF. Influence of contrast agent dose and ultrasound exposure on cardiomyocyte injury induced by myocardial contrast echocardiography in rats. Radiology. 2005; 237:137-143. [PubMed: 16183929]

15. Miller DL, Dou CY, Lucchesi BR. Are Ecg Premature Complexes Induced by Ultrasonic Cavitation Electrophysiological Responses to Irreversible Cardiomyocyte Injury? Ultrasound Med Biol. Feb.2011 37:312-320. [PubMed: 21257092]

16. Weissleder R. Scaling down imaging: molecular mapping of cancer in mice. Nat Rev Cancer. Jan. 2002 2:11-8. [PubMed: 11902581]

17. Weissleder R. Molecular imaging in cancer. Science. May 26.2006 312:1168-71. [PubMed: 16728630]

18. Eils R, Athale C. Computational imaging in cell biology. J Cell Biol. May 12.2003 161:477-81. [PubMed: 12743101]

19. Pham DL, Xu C, Prince JL. Current methods in medical image segmentation. Annu Rev Biomed Eng. 2000; 2:315-37. [PubMed: 11701515]

20. Baker CJ, Mock NM. An Improved Method for Monitoring Cell-Death in Cell-Suspension and Leaf Disc Assays Using Evans Blue. Plant Cell Tiss Org. Oct.1994 39:7-12.

21. Bassien-Capsa V, Fouron JC, Comte B, Chorvatova A. Structural, functional and metabolic remodeling of rat left ventricular myocytes in normal and in sodium-supplemented pregnancy. Cardiovasc Res. Feb 1.2006 69:423-431. [PubMed: 16337612]

22. Ho SY. Anatomy and myoarchitecture of the left ventricular wall in normal and in disease. Eur J Echocardiogr. Dec.2009 10:iii3-7. [PubMed: 19889656]

23. Qu, F.; Ren, D.; Liu, X.; Jing, Z.; Yan, L. A face image illumination quality evaluation method based on Gaussian low-pass filter. Cloud Computing and Intelligent Systems (CCIS), 2012 IEEE 2nd International Conference on; 2012; p. 176-180. 

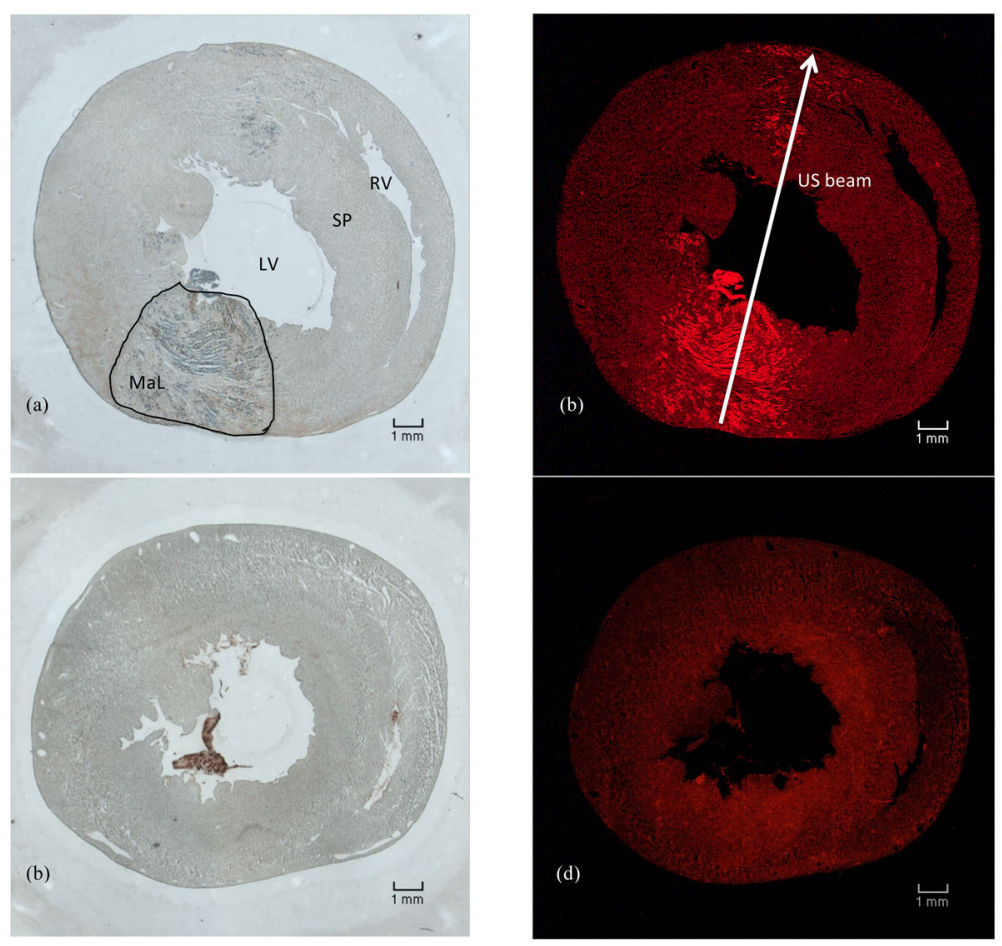

Fig. 1.

Evans blue staining indicated injured cardiomyocytes for microscope assessment as shown in (a) within an area noted by MaL (macrolesion), where also LV (left ventricle), RV (right ventricle) and SP (septum) are labeled. An example tissue section of treated heart muscle is shown in brightfield (a) and fluorescence (b). The damaged cardiomyocytes in scattered microlesions appear stained light blue in the brightfield image, together with erythrocytes and other cells associated with the co-located microvascular hemorrhage, as shown (a). The damaged cardiomyocytes specifically appear fluorescent red in the relatively high contrast fluorescence image as shown in (b). The therapeutic ultrasound beam indicated by the white arrow in (b) is estimated from the lesion cloud as described in the text. (US: ultrasound). Similarly, the brightfield image (c) and the fluorescence image (d) of an example tissue section of a sham case are shown. The background fluorescence includes a few bright spots due to cell overlap, arterial walls and other autofluorescent normal structures. 


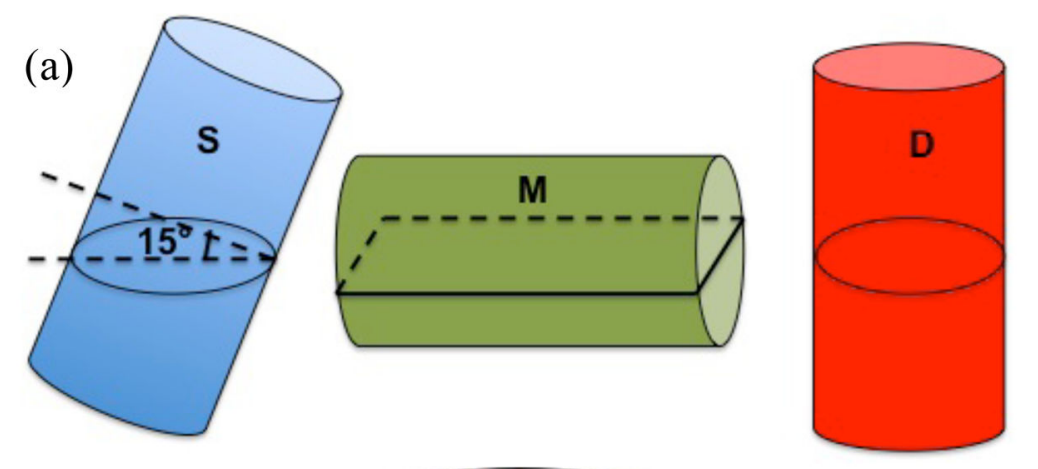

(b)

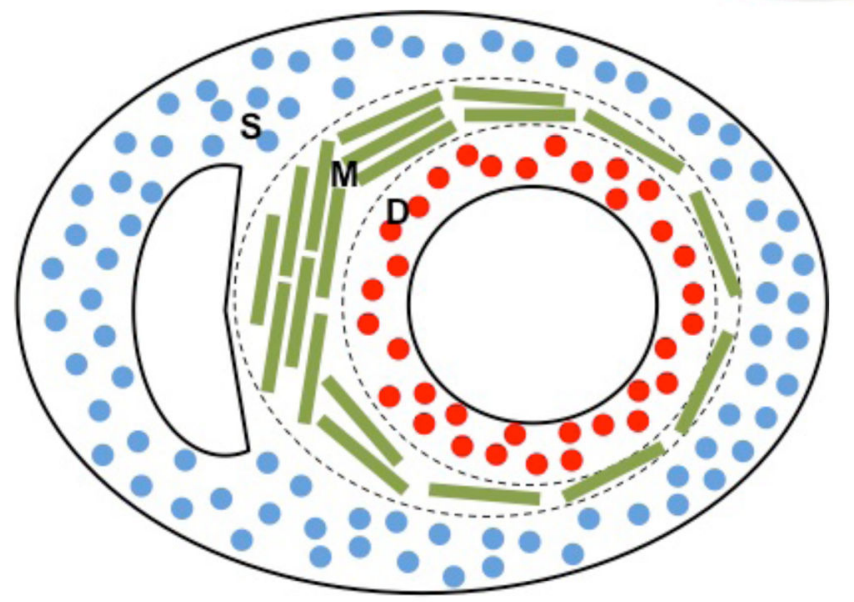

Fig. 2.

Illustration of a tissue slice plane intersecting with different layers of the ventricular wall:

(a) Schematic diagram for three elliptic-cylinder modeled cardiomyocytes oriented differently for each layer: blue for superficial (S), green for middle (M) and red for deep (D) layer; (b) Schematic diagram for the orientation of cardiomyocytes in each layer: blue for superficial (S), green for middle (M) and red for deep (D) layer. 


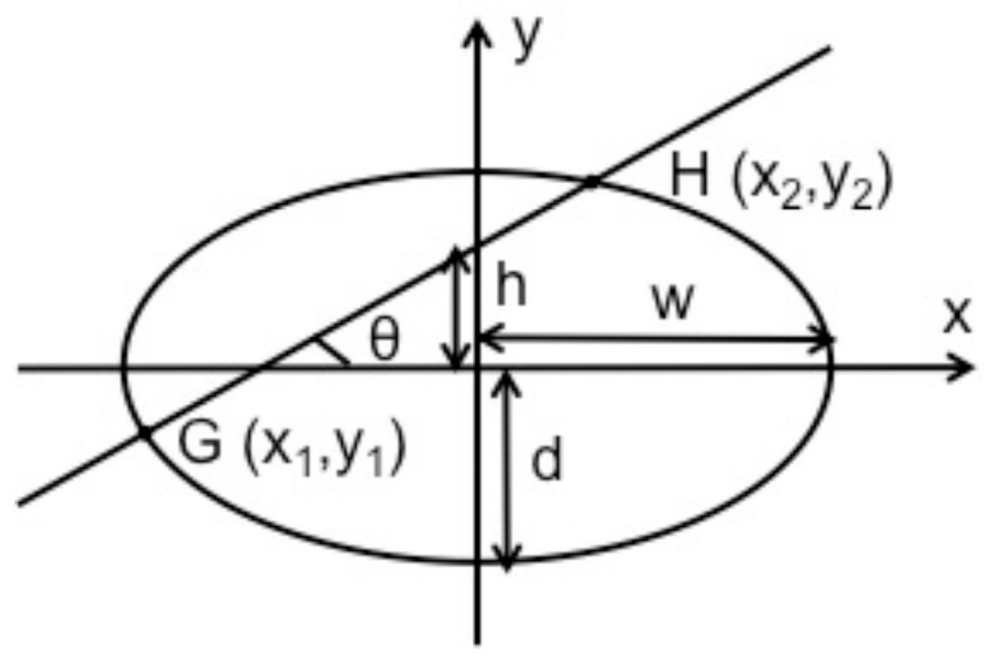

Fig. 3.

Top view of an elliptic cylinder cell intersected with the cutting plane at an angle of $\theta$. The cutting edge is $\mathrm{GH}$. 


\begin{tabular}{|c|c|c|c|c|}
\hline $\begin{array}{l}\text { Step 1: } \\
\text { Tissue }\end{array}$ & $\begin{array}{c}\text { Step 2: } \\
\text { Microlesion }\end{array}$ & $\begin{array}{c}\text { Step 3: } \\
\text { Image Registration }\end{array}$ & $\begin{array}{l}\text { Step 4: } \\
\text { Therapeutic Beam }\end{array}$ & $\begin{array}{c}\text { Step 5: } \\
\text { Macrolesion }\end{array}$ \\
\hline Detection & Detection & \& 3D Stacking & Estimation & Characterization \\
\hline
\end{tabular}

Fig. 4.

Flow chart for the overall method of macrolesion characterization. 

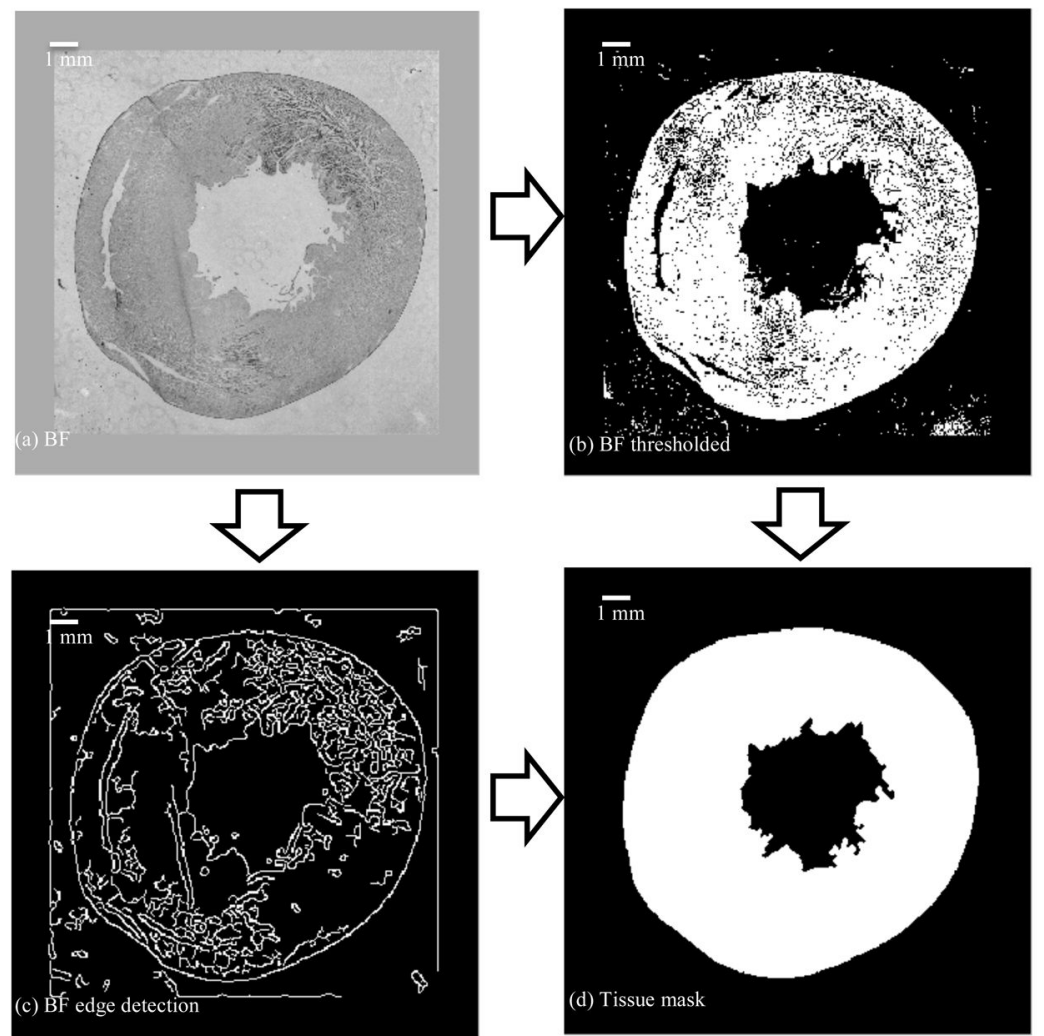

Fig. 5.

A threshold mask as shown in (b) and an edge-detected mask as shown in (c) were obtained simultaneously from a normalized brightfield image as shown in (a). Fusion of these resulted in the tissue mask as shown in d). 

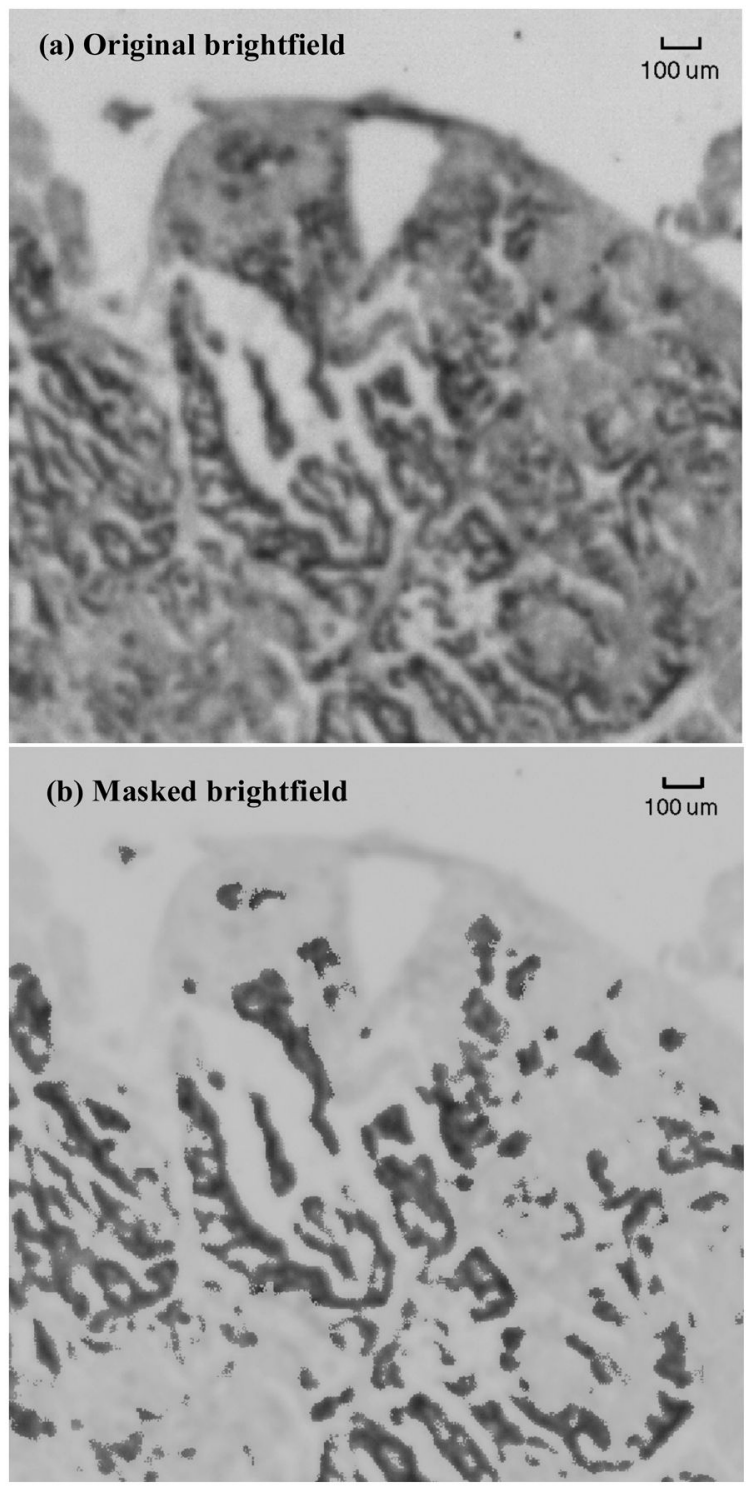

Fig. 6.

An automatic threshold for fluorescence image has been used to detect microlesions shown masked on the brightfield image in (b) compared to original brightfield image in (a). Note: images were zoomed in. 


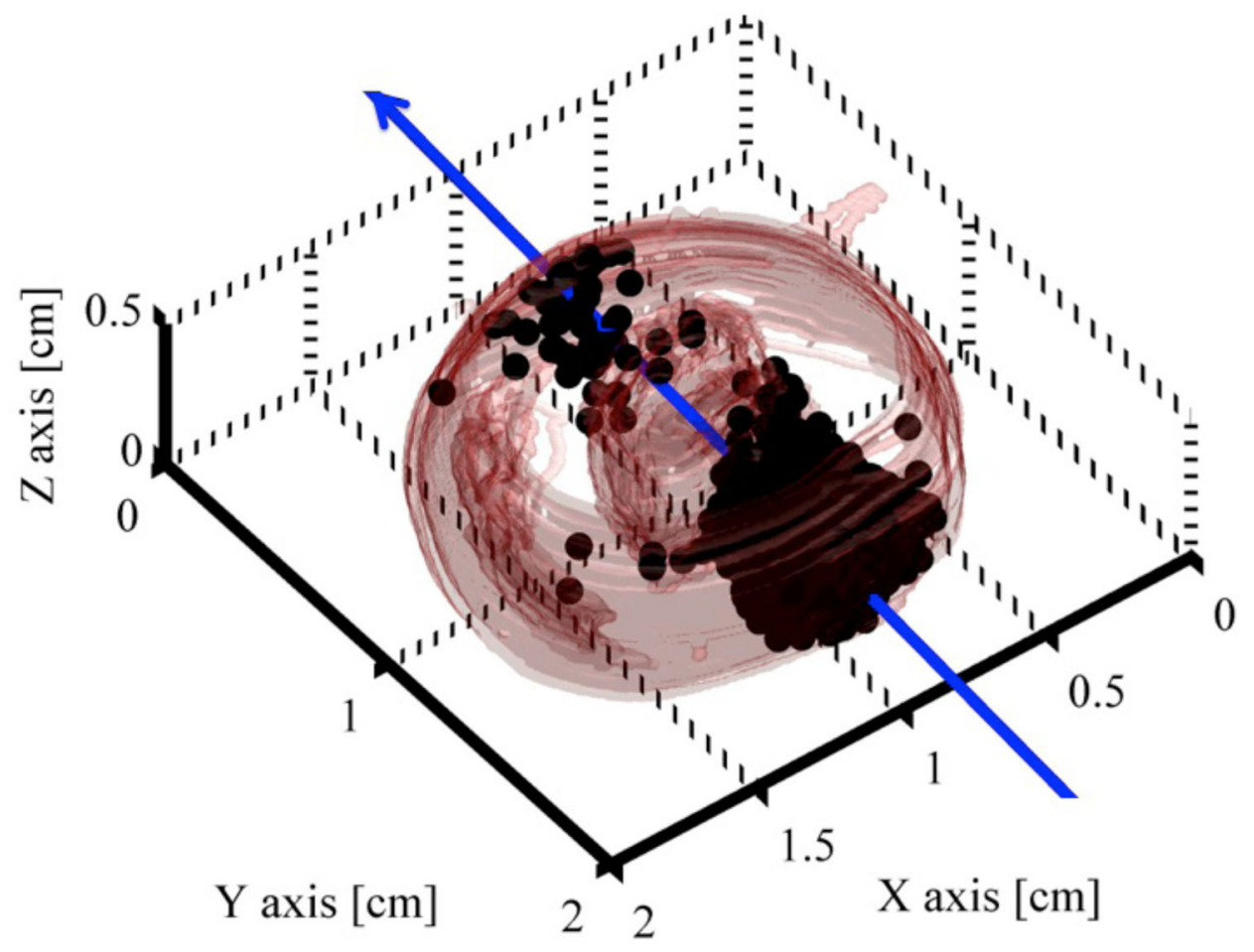

Fig. 7.

Thee-dimensional stacking of registered image masks allows for visualization of the employed 3D model, where the red heart tissue surface outline is rendered as a result from step 1 (tissue detection), black marks symbolize downsampled microlesions detected in step 2 and the therapeutic ultrasound beam (blue line) is characterized from least square fitting of the microlesion cloud (see text for more details). 

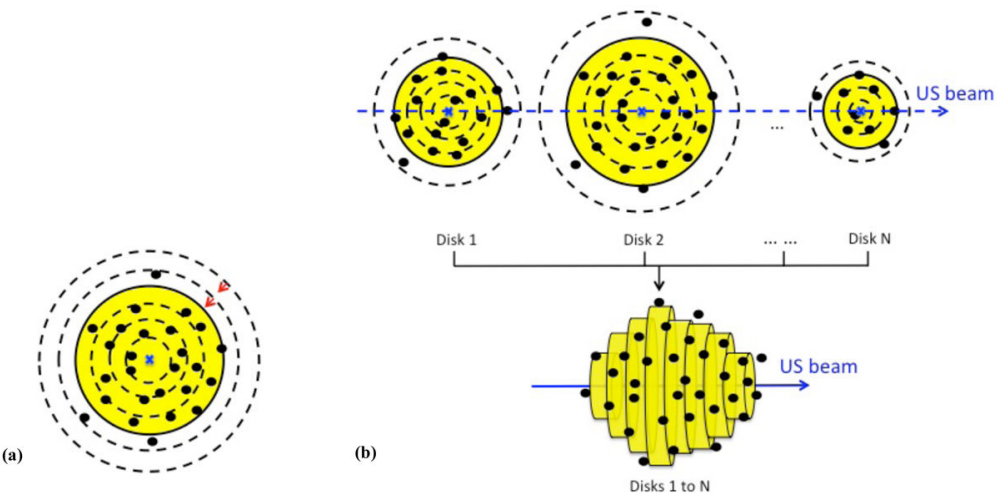

Fig. 8.

Schematic diagram of the procedure for macrolesion characterization. The stacked 3D heart model was re-sliced along the therapeutic beam in $800 \mu \mathrm{m}$-thick steps. At each position along the beam axis noted by blue $\mathrm{x}$, a disk was characterized as illustrated in (a): each such slice was first populated with a $20 \mathrm{~mm}$-diameter disk coaxial to the beam identified as the most outer dash circle; each disk was then progressively reduced in size as the red arrow implies until a certain microlesion quantity was reached from a dynamic threshold, i.e. ends up with a characterized disk shown as yellow surrounded by solid line. The yellow cylinderlike volume as in (b), i.e. stacked disks, is defined as a macrolesion, characterized by a dynamic thresholding algorithm for counting enclosed microlesions for each disk step as illustrated in (a). (US: ultrasound) 


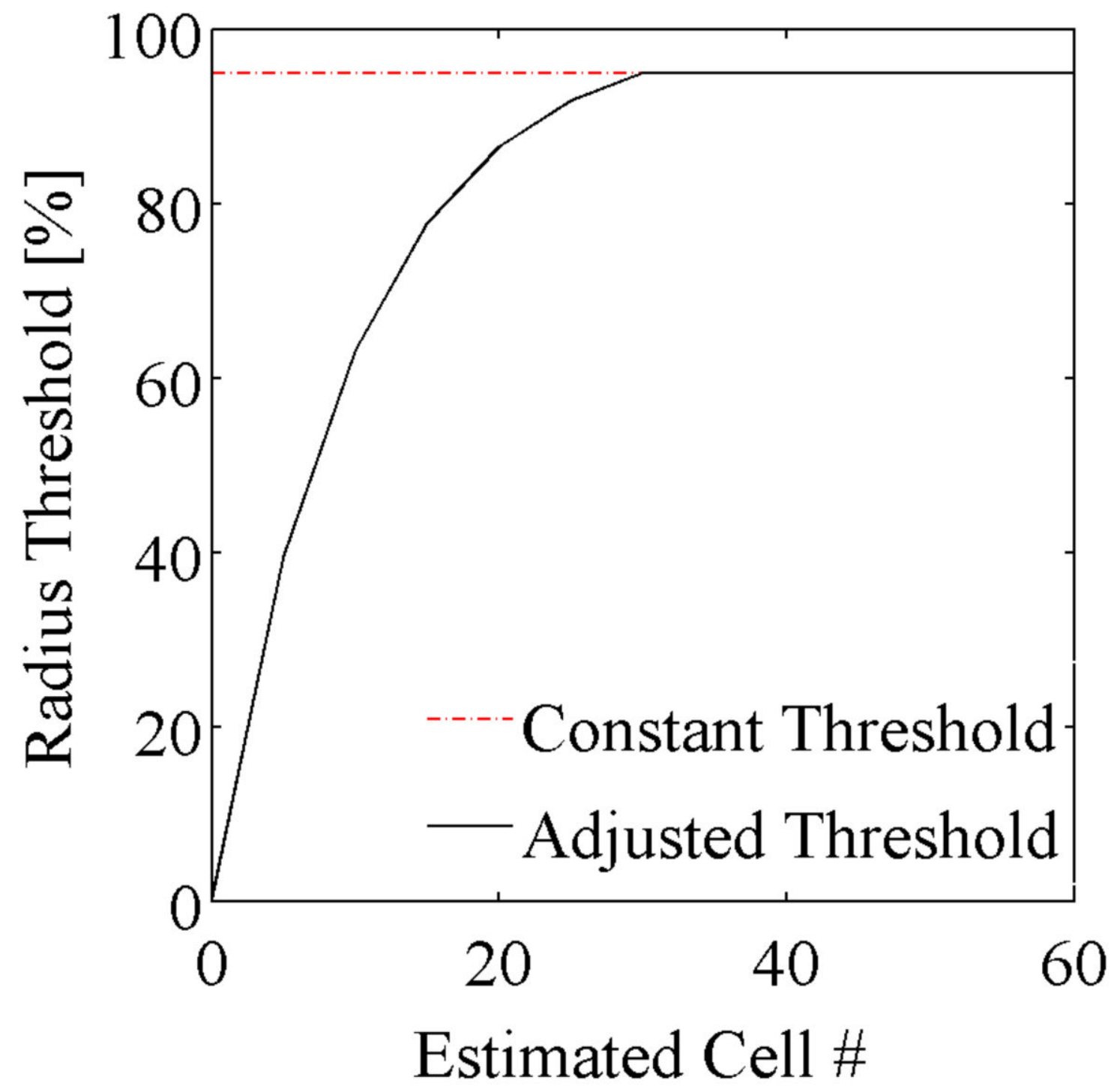

Fig. 9.

For a stained cell number cut-off threshold of 30 the auto-adjusted threshold is plotted as a function of counted stained cells. If only 20 stained cells are counted within the current axial slice, then the macrolesion radius is set such that approximately $80 \%$ of the counted stained cells are enclosed. 


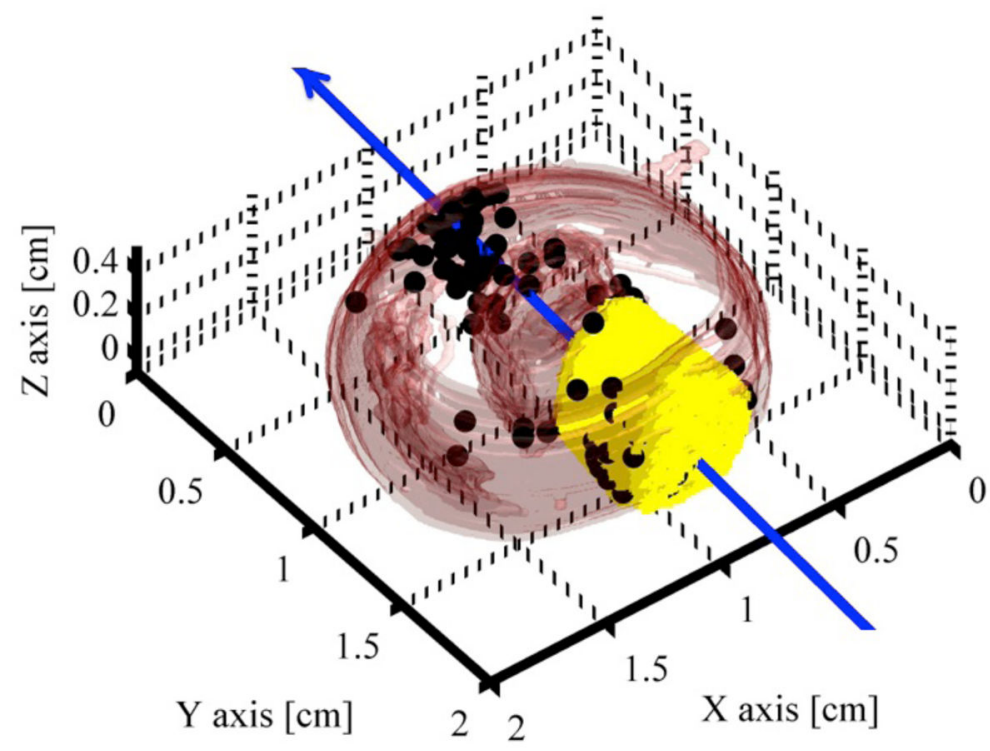

Fig. 10.

Characterized macrolesion visualization: same as Fig. 7, additionally the resulting volumetric macrolesion is shown as yellow disks along the therapeutic beam. Note that it did not conclude the distal cloud of microlesions as one part of macrolesion because the criteria was customized to reject volume with less than $10 \%$ microlesion density, which is to be explored later in long-term study that how much is the microlesion density to cause significant tissue shrinkage. 

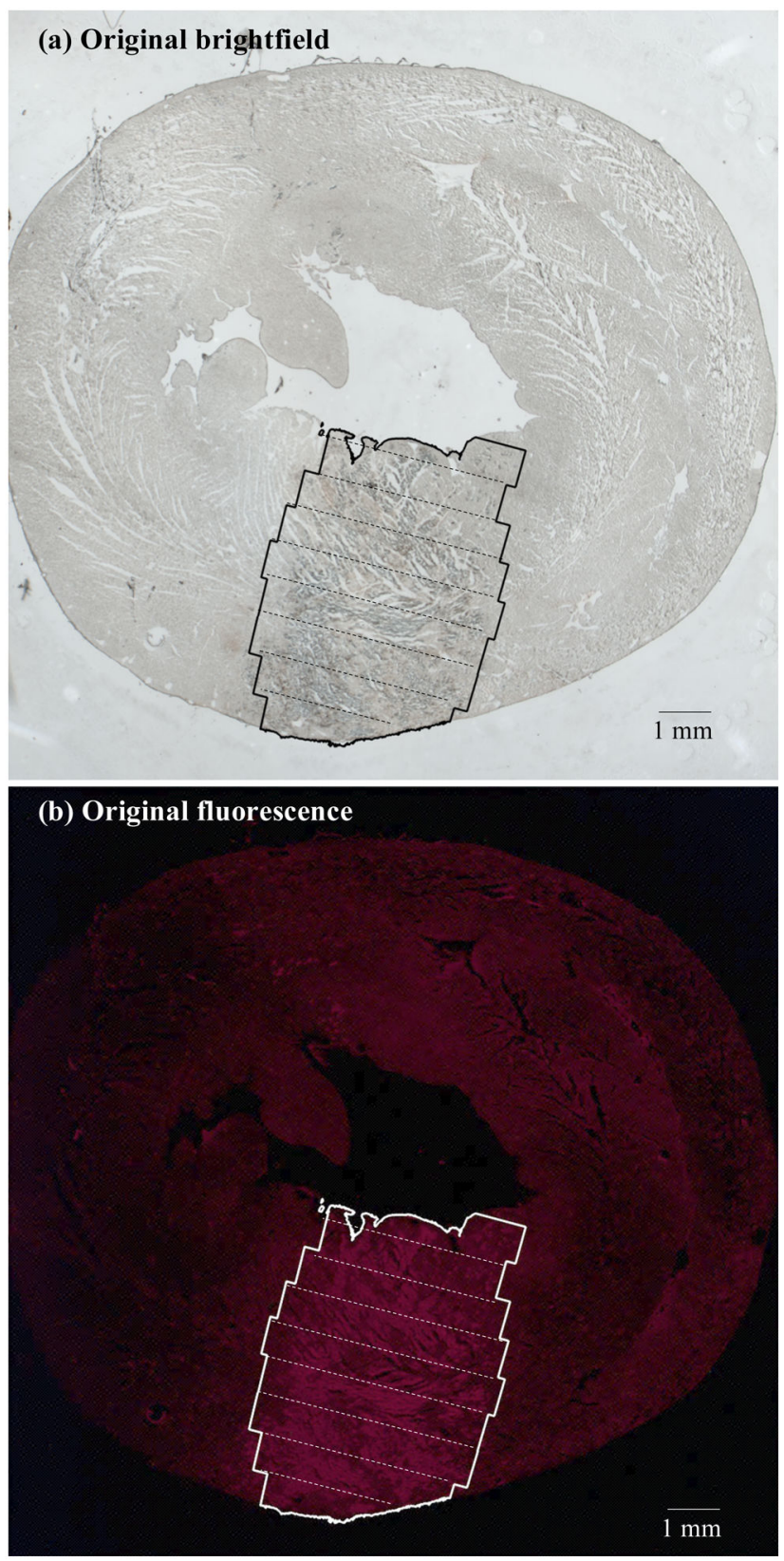

Fig. 11.

An example set of images showing the characterized macrolesion projected back onto its original 2D microscopy images with dashed lines indicating stacked cross-sectioned cylinders and tissue boundary derived from brightfield tissue detection. 

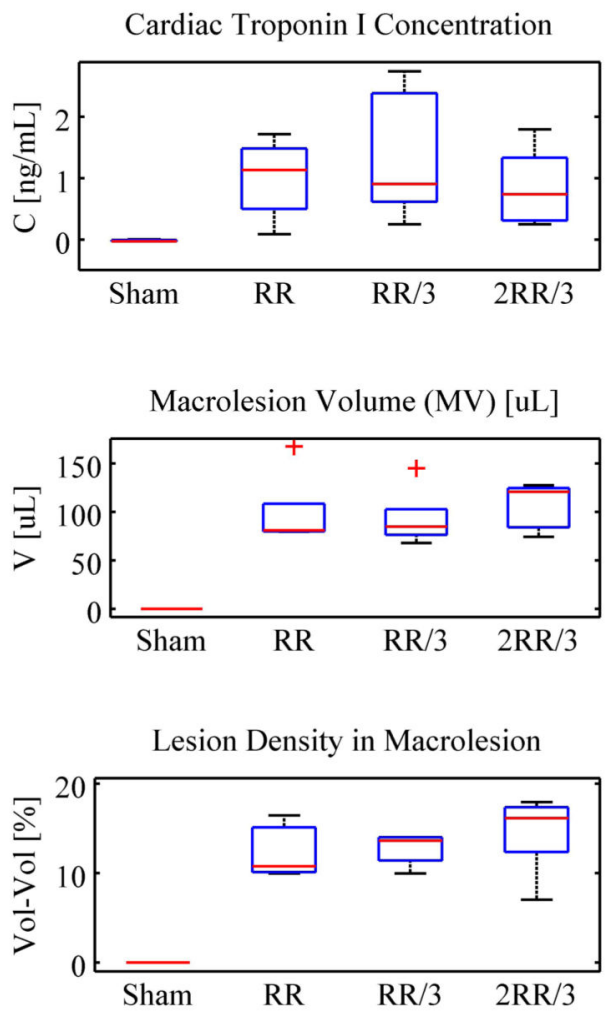

Mean Radius of Macrolesion

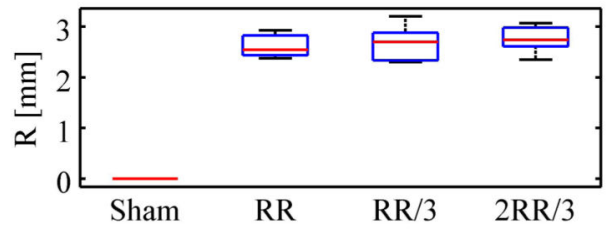

Length of Macrolesion

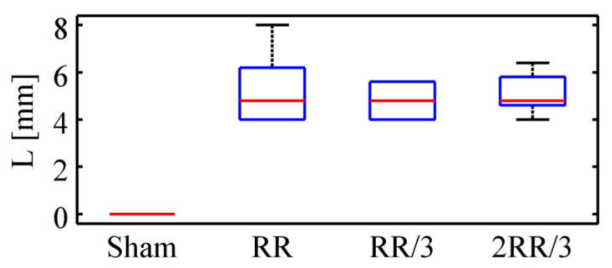

Fig. 12.

Characterized features of macrolesions are compared to the measurement of troponin I in plasma samples taken one day after the treatment. The treated group was subdivided into 3 subgroups being treated at different time points of the cardiac cycle: $R$ wave (RR, end diastole), $\mathrm{R}$ wave plus $1 / 3$ of the $\mathrm{R}$ to $\mathrm{R}$ interval ( $\mathrm{RR} / 3$, end systole) and $\mathrm{R}$ wave plus $2 / 3$ of the $\mathrm{R}$ to $\mathrm{R}$ interval (2RR/3, mid-diastole). 

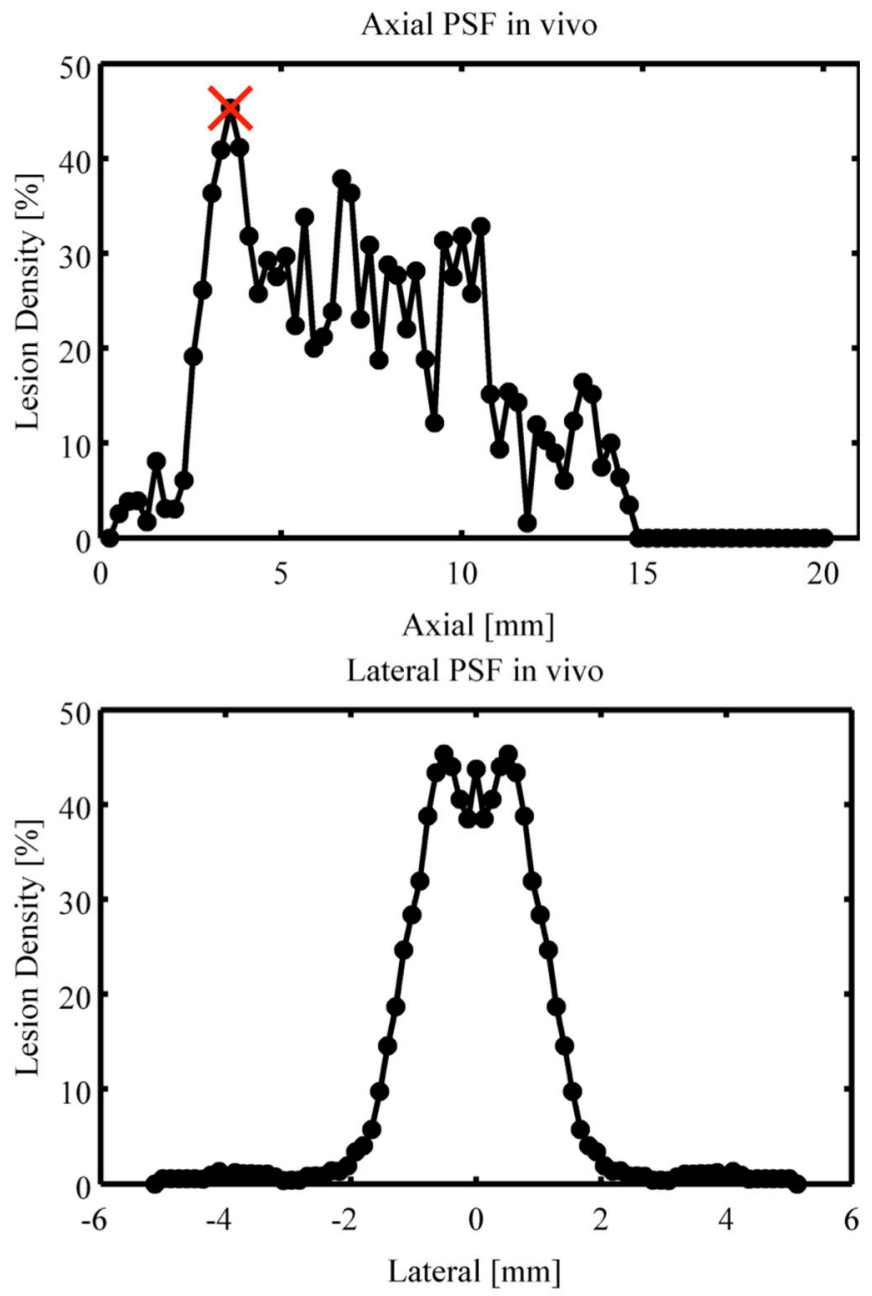

Fig. 13.

The in vivo point spread function (PSF) of the therapeutic beam as assessed in terms of lesion density along the axis of the treatment transducer. Left: macrolesion along the therapeutic beam axis; right: lateral cut through macrolesion at axial maximum (indicated as an ' $x$ ' on the left). 

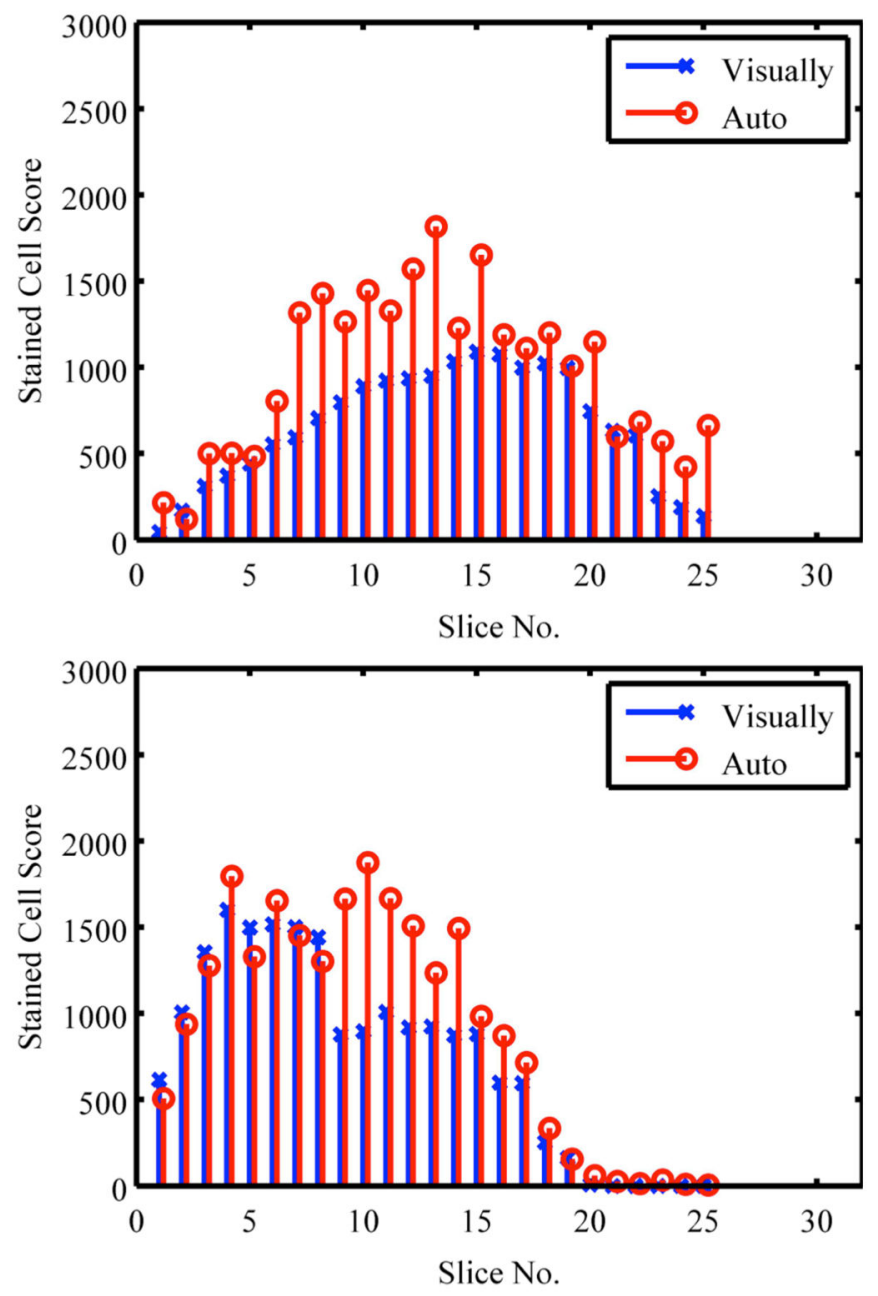

Fig. 14.

Two examples of comparison on visual cell scoring and automatic scoring derived from the proposed computer-based quantification method with cardiomyocyte model. The proposed method gives an area-based estimation of stained cell number instead of a subjective judgment based on microlesion morphology. 


\section{TABLE I}

Single cardiomyocyte geometry model

\begin{tabular}{lccc}
\hline Parameter & Abbreviation & Value & Unit \\
\hline Cell Length & $l$ & $113.4 \pm 0.8$ & $\mu \mathrm{m}$ \\
Cell Width & $w$ & $28.9 \pm 0.4$ & $\mu \mathrm{m}$ \\
Cell Depth & $d$ & $17.6 \pm 0.4$ & $\mu \mathrm{m}$ \\
\hline
\end{tabular}




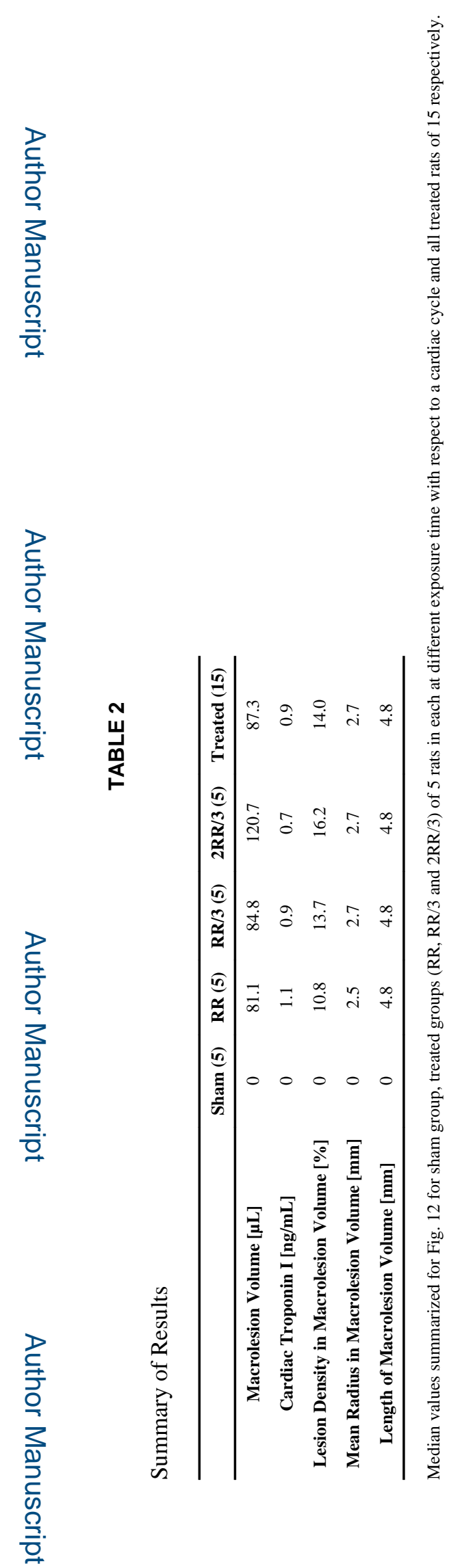

IEEE Trans Biomed Eng. Author manuscript; available in PMC 2015 May 19. 